\title{
Biased total mass of cool core galaxy clusters by Sunyaev-Zel'dovich effect measurements
}

\author{
A. Conte, M. De Petris, B. Comis, L. Lamagna, and S. De Gregori
}

Sapienza University, Department of Physics, Piazzale Aldo Moro 2, 000185 Rome, Italy

e-mail: andrea.conte@roma1.infn.it

Received 2 March 2011 / Accepted 5 May 2011

\section{ABSTRACT}

\begin{abstract}
The Sunyaev Zel'dovich (SZ) effect from galaxy clusters is one of the most powerful cosmological tools for investigating the largescale Universe. The big advantage of the SZ effect is its redshift independence, which is not the case for visible and X-ray observations. It allows us to directly estimate the cluster's total mass from the integrated comptonization parameter $Y$, even for distant clusters. However, not having a full knowing intra-cluster medium (ICM) physics can affect the results. By taking self-similar temperature and density profiles of the ICM into account, we studied how different ICM morphologies can affect the cluster total mass estimation. With the help of the high percentage of cool core (CC) clusters, as observed so far, the present analysis focuses on studying this class of objects. A sample of eight nearby $(0.1<z<0.5)$ and high-mass $\left(M>10^{14} M_{\odot}\right)$ clusters observed by Chandra was considered. We simulated SZ observations of these clusters through X-ray derived information and analyzed the mock SZ data again with the simplistic assumption of an isothermal beta-model profile for the ICM. The bias on the recovered cluster total mass using different sets of assumptions is estimated to be $50 \%$ higher in the case of hydrostatic equilibrium. Possible contributions to the total bias due to the line-of-sight integration and the considered ICM template are taken into account. The large biases on total mass recovery firmly support, if still necessary, cluster modeling based on more sophisticated universal profiles as derived by X-ray observations of local objects and hydrodynamical simulations.
\end{abstract}

Key words. galaxies: clusters: general - cosmic backgroud radiation

\section{Introduction}

Clusters of galaxies are the largest gravitationally-bound objects arising thus far from the process of hierarchical structure formation (Voit 2005). As the most recent and most massive objects of the Universe, clusters are excellent probes for studying its formation and evolution. The observed state of gas within a cluster is determined by a combination of shock heating during accretion, radiative cooling, and thermal feedback produced by the cooling itself, so the density and temperature of the ICM represent the full thermal history of clusters' formation. To better understand the physics of ICM, it is necessary to have sufficient knowledge of the gas density and temperature distributions. Though clusters are the ideal target objects for X-ray observations of the hot ICM, millimeter and sub-millimeter measurements provide independent and complementary tools for studying the same ICM by exploiting the Sunyaev Zel'dovich (SZ) effect (Sunyaev \& Zel'dovich 1972).

The SZ effect is the Comptonization of the cosmic microwave background (CMB) photons, coming from the last scattering surface, by the hot electrons population of the ICM. The photon energy variation, which is caused by the scattering process, can be expressed as CMB temperature variations

$\Delta T_{\mathrm{SZ}}=y T_{\mathrm{CMB}} f(x)\left(1+\delta_{n}\left(x, \theta_{\mathrm{e}}\right)\right)+\Delta T_{\text {kin }}$

where

$y=\int \theta_{\mathrm{e}} \mathrm{d} \tau_{\mathrm{e}}=\int\left(\frac{k_{\mathrm{B}} T_{\mathrm{e}}}{m_{\mathrm{e}} c^{2}}\right) \sigma_{\mathrm{T}} n_{\mathrm{e}} \mathrm{d} l \propto \int P_{\mathrm{e}} \mathrm{d} l$

represents the comptonization parameter, $x=(h v) /\left(k_{\mathrm{B}} T_{\mathrm{CMB}}\right)$ the dimensionless frequency, $h$ and $k_{\mathrm{B}}$ are respectively the Planck and Boltzmann constants, $T_{\mathrm{CMB}}, m_{\mathrm{e}}$ and $\sigma_{\mathrm{T}}$, the CMB temperature at $z=0$, the electron mass at rest and the Thomson cross section, $\theta_{\mathrm{e}}$ represents the dimensionless thermal energy of the ICM, $\tau_{\mathrm{e}}$ is the electron optical depth. The parameters $n_{\mathrm{e}}$, $T_{\mathrm{e}}$, and $P_{\mathrm{e}}$ are the electron number density, temperature, and pressure of the ICM, $\delta_{n}\left(x, \theta_{\mathrm{e}}\right)=f_{n}(x) \theta_{\mathrm{e}}^{n} / f(x)$ is the relativistic correction term that accounts for the thermal energy of the electrons involved in the scattering processes, where $f(x)=$ $x\left[\left(\mathrm{e}^{x}+1\right) /\left(\mathrm{e}^{x}-1\right)-4\right]$ is a dimensionless quantity that describes the spectral signature of the effect, and the subscript $n$ indicates the maximum order of the relativistic correction $(n=4$ in this work, Nozawa et al. 2005). The last term of Eq. (1) is the kinematic component of the SZ effect, which contains the contribution from the bulk motion of the electron population with respect to the last scattering surface reference frame. For the purpose of this paper, this term is omitted, assuming that it is disentangled from the thermal component by multi-frequency observations, together with the signal from the primary CMB emission.

The SZ effect is redshift independent and, for this reason, it is possible to detect distant clusters without any existing $\mathrm{X}$-ray or optical observations. This is the case of the ongoing ground-based experiments such as SPT (Ruhl et al. 2004), ACT (Kosowsky et al. 2003), and the all sky survey like Planck (Planck Collaboration 2011a) or the upgraded MITO (De Petris et al. 2007) and OLIMPO (Masi et al. 2008) with new spectroscopic capabilities and the proposed 30-m diameter C-CAT (Sebring et al. 2006). However, some assumptions on cluster physics still have to be made in order to directly extract cluster observables.

Estimates of clusters' total mass can be derived by SZ observations when X-ray or lensing measurements are available or by 
empirically calibrated scaling relations linking the SZ flux to the total mass (e.g. Vikhlinin et al. 2009; Arnaud et al. 2010; Planck Collaboration 2011b; Comis et al. 2011). Total mass can also be determined by SZ observations alone when applying thermal energy constraints (Mroczkowski et al. 2011).

To accurately reproduce the gas inside the cluster, an ICM universal model is mandatory (e.g. Nagai et al. 2007; Arnaud et al. 2010). In this paper we confirm that the simple isothermal beta-model is clearly an inappropriate cluster representation for total mass recovery by SZ observations, particularly in the presence of relaxed cool core (CC) clusters. These objects show a well studied peaked density profile with a temperature decrement in the core region (Jones \& Forman 1984). In the local universe this class of clusters is observationally a significant percentage of the total cluster population (Eckert et al. 2011). Even if the X-ray estimated CC fraction is biased by selection effects in flux-limited samples, recently a $35 \%$ of clusters have picked up in the SZ high signal-to-noise ratio Planck early cluster data-set (Planck Collaboration 2011c) are CC clusters. Large scatter in mass estimates of CC clusters has been highlighted previously using numerical simulations by Hallman et al. (2006) and Hallman et al. (2007).

We investigate the bias on the mass in a limited sample of eight nearby $(0.1<z<0.5)$ and high-mass $\left(M>10^{14} M_{\odot}\right) \mathrm{CC}$ clusters observed by Chandra. The SZ maps of these clusters, which are expressed in thermodynamic temperature units and convolved with several instrumental beams, are dealt with by applying the isothermal beta-model. The total mass is derived in three different ways: by assuming hydrostatic equilibrium and a fixed gas fraction and by applying a self similar scaling relation. To focus only on the consequences of the employed ICM model, in our analysis we neglect all the contaminants present in the sky by assuming in this way the best situation to recover cluster total mass.

In Sect. 2, we discuss the electron number density radial profile and follow self-similar studies to characterize a universal electron temperature radial profile of a limited sample of eight CC clusters observed by Chandra. In Sect. 3 we generate maps of the SZ effect in thermodynamic temperature. In Sect. 4 we evaluate cluster total mass under different sets of assumptions. The bias on the recovered mass is described in Sect. 5, which discusses the main contributions. Conclusions are summarized in Sect. 6.

\section{Electron number density and temperature profiles}

A general parametric model for the cluster atmosphere must be defined to forecast the shape of cluster SZ signals in matched filter techniques for detecting clusters in blind surveys. ACT has detected new clusters assuming a two-dimensional Gaussian profile as filter (Sehgal et al. 2011), while SPT has detected a projected spherical beta profile (Vanderlinde et al. 2010) and Planck a universal pressure profile (Melin et al. 2011).

The approach for determining the total mass cluster can be different. High-quality X-ray data allows an accurate modeling of cluster morphology, but in the case of low angular resolution and/or low signal-to-noise ratio a simple isothermal beta-model is still applied (e.g. Marriage et al. 2010; Sayers et al. 2011).

In this work we analyze this model (Cavaliere \& Fusco-Femiano 1978), which is based on the very general assumption that the electron temperature $T_{\mathrm{e}}$ is constant along the whole considered cluster radial extension and that the electron number density follows a spherical distribution as

$n_{\mathrm{e}, \mathrm{ISO}}(r)=n_{\mathrm{e} 0}\left(1+\frac{r^{2}}{r_{\mathrm{c}}^{2}}\right)^{-\frac{3}{2} \beta}$

where $n_{\mathrm{e} 0}$ is the central electron number density, $r_{\mathrm{c}}$ the core radius, and $\beta$ the power law index. The subscript ISO indicates, hereafter, the isothermal beta-model. The proved inadequacy of this model is compensated for by the advantage of extracting a simple analytic expression for the $y$ parameter along the off-axis angular separation, $\theta$,

$y_{\mathrm{ISO}}(\theta)=y_{0}\left(1+\frac{\theta^{2}}{\theta_{\mathrm{c}}^{2}}\right)^{\frac{1}{2}-\frac{3}{2} \beta}$

where

$y_{0}=n_{\mathrm{e} 0} \frac{k_{\mathrm{B}} T_{\mathrm{e}}}{m_{\mathrm{e}} c^{2}} \sigma_{\mathrm{T}} r_{\mathrm{c}} \sqrt{\pi} \frac{\Gamma\left(\frac{3}{2} \beta-\frac{1}{2}\right)}{\Gamma\left(\frac{3}{2} \beta\right)}$,

and $\theta_{\mathrm{c}}=r_{\mathrm{c}} / D_{\mathrm{A}}$, with $D_{\mathrm{A}}$ the angular diameter distance, which has been calculated for each cluster by using

$D_{\mathrm{A}}=\frac{c}{H_{0}(1+z)} \int_{0}^{z} \frac{\mathrm{d} z^{\prime}}{E\left(z^{\prime}\right)}$,

where $H_{0}$ is the Hubble constant and $E(z)=$ $\left[\Omega_{\mathrm{M}}(1+z)^{3}+\Omega_{\Lambda}\right]^{1 / 2}$. We adopt a $\Lambda C D M$ cosmology with $H_{0}=70 \mathrm{~km} \mathrm{~s}^{-1} \mathrm{Mpc}^{-1}, \Omega_{\mathrm{M}}=0.3$, and $\Omega_{\Lambda}=0.7$.

It is easy to find clusters that are not relaxed or that display structures that are very difficult to describe with this model. Therefore, it is realistic to assume that many newly discovered clusters in blind SZ surveys exhibit such significant deviations as well. To explore a particular ICM gas morphology, we focus on CC clusters. We started analyzing a small sample of eight objects extracted from the Chandra dataset investigated in Bonamente et al. (2006).

The study of a central region, commonly known as the core region, has been challenged by high-resolution numerical simulations (Navarro et al. 1995; Borgani et al. 2004; Kay et al. 2004; Nagai et al. 2007; Henning et al. 2009). These works lead to an agreement on whether there is a cooling core in the very central denser gas region $\left(r<0.1 r_{500}\right)$ of some clusters, as well as a slower decline in the temperature at large radii $\left(r>0.2 r_{500}\right)$. As usual we refer to $r_{500}$ as the radius of the cluster that defines a volume with mean density 500 times the critical density $\rho_{\text {crit }}$ at cluster redshift. The choice of $r_{500}$ is motivated by simulation results from Evrard et al. (1996) showing the gas within this radius relaxed and in hydrostatic equilibrium.

Moreover, many observational studies in X-rays have shown that the X-ray surface brightness, hence the underlying density, cannot be represented correctly by a beta profile. A second component should be added or a peaked central part introduced in order to properly fit the observation. The observed deprojected density profiles are peaked for CC systems and flatter for morphologically disturbed clusters.

The $n_{\mathrm{e}}$ cluster profile can be described by a double betamodel profile

$n_{\mathrm{e}, \mathrm{CC}}(r)=n_{\mathrm{e} 0}\left[f\left(1+\frac{r^{2}}{r_{\mathrm{c} 1}^{2}}\right)^{-\frac{3}{2} \beta}+(1-f)\left(1+\frac{r^{2}}{r_{\mathrm{c} 2}^{2}}\right)^{-\frac{3}{2} \beta}\right]$,

where the parameters' data have been taken from Bonamente et al. (2006) and adapted to this work to have a symmetric standard deviation (D’Agostini 2003). 
Table 1. Parameters of the electron number density profiles.

\begin{tabular}{lccccc}
\hline \hline Cluster & $\begin{array}{c}n_{\mathrm{e} 0} \\
\left(10^{-2} \mathrm{~cm}^{-3}\right)\end{array}$ & $\beta$ & $\begin{array}{c}\theta_{\mathrm{c} 1} \\
(\operatorname{arcsec})\end{array}$ & $\begin{array}{c}\theta_{\mathrm{c} 2} \\
(\operatorname{arcsec})\end{array}$ & $f$ \\
\hline A1413 & $3.89 \pm 0.54$ & $0.535 \pm 0.016$ & $6.7 \pm 1.4$ & $40.1 \pm 4.1$ & $0.760 \pm 0.020$ \\
A1689 & $4.15 \pm 0.31$ & $0.871 \pm 0.040$ & $21.6 \pm 1.0$ & $104.5 \pm 5.3$ & $0.870 \pm 0.010$ \\
A1835 & $11.3 \pm 0.4$ & $0.802 \pm 0.015$ & $9.3 \pm 0.2$ & $63.8 \pm 1.6$ & $0.940 \pm 0.001$ \\
A2204 & $20.4 \pm 1.1$ & $0.716 \pm 0.028$ & $7.5 \pm 0.3$ & $67.6 \pm 1.9$ & $0.959 \pm 0.004$ \\
A2261 & $4.07 \pm 0.59$ & $0.631 \pm 0.024$ & $10.2 \pm 1.8$ & $39.1 \pm 5.9$ & $0.760 \pm 0.050$ \\
MS1358.4+6245 & $9.63 \pm 0.79$ & $0.676 \pm 0.017$ & $3.3 \pm 0.2$ & $37.0 \pm 1.8$ & $0.934 \pm 0.003$ \\
RXJ1347.5-1145 & $28.5 \pm 1.4$ & $0.632 \pm 0.009$ & $4.0 \pm 0.2$ & $23.3 \pm 1.6$ & $0.942 \pm 0.004$ \\
ZW3146 & $16.9 \pm 0.3$ & $0.669 \pm 0.005$ & $4.4 \pm 0.1$ & $25.8 \pm 0.6$ & $0.882 \pm 0.004$ \\
\hline
\end{tabular}

Table 2. CC galaxy clusters properties used in the analysis to generate a universal $T_{\mathrm{e}}$ profile for this class of clusters.

\begin{tabular}{lcccccc}
\hline \hline $\begin{array}{l}\text { Cluster } \\
\text { name }\end{array}$ & $z$ & $\begin{array}{c}D_{\mathrm{A}} \\
(\mathrm{Gpc})\end{array}$ & $\begin{array}{c}r_{500}{ }^{a} \\
(\mathrm{kpc})\end{array}$ & $\begin{array}{c}\theta_{500} \\
(\mathrm{arcsec})\end{array}$ & $\begin{array}{c}T_{\mathrm{X}}{ }^{b} \\
(\mathrm{keV})\end{array}$ & $\begin{array}{c}T_{\mathrm{e} 0}{ }^{c} \\
(\mathrm{keV})\end{array}$ \\
\hline A1413 & 0.142 & 0.52 & $1195 \pm 232$ & $321 \pm 62$ & $6.6 \pm 0.6$ & $7.3 \pm 0.2$ \\
A1689 & 0.183 & 0.63 & $1402 \pm 260$ & $377 \pm 70$ & $8.7 \pm 0.9$ & $10.0 \pm 0.3$ \\
A1835 & 0.252 & 0.81 & $1439 \pm 414$ & $387 \pm 111$ & $10.5 \pm 1.0$ & $8.4 \pm 0.2$ \\
A2204 & 0.152 & 0.55 & $1796 \pm 320$ & $483 \pm 86$ & $11.3 \pm 1.8$ & $6.5 \pm 0.2$ \\
A2261 & 0.224 & 0.74 & $1201 \pm 168$ & $323 \pm 45$ & $7.0 \pm 1.0$ & $7.2 \pm 0.4$ \\
MS1358.4+6245 & 0.327 & 0.97 & $1633 \pm 885$ & $439 \pm 238$ & $8.4 \pm 1.1$ & $8.3 \pm 0.6$ \\
RXJ1347.5-1145 & 0.451 & 1.19 & $1734 \pm 170$ & $466 \pm 46$ & $14.8 \pm 1.5$ & $13.5 \pm 0.5$ \\
ZW3146 & 0.291 & 0.90 & $1804 \pm 344$ & $485 \pm 92$ & $8.7 \pm 0.4$ & $6.6 \pm 0.1$ \\
\hline
\end{tabular}

Notes. ${ }^{(a)}$ Morandi et al. (2007). ${ }^{(b)}$ Temperature scale calculated by a weighted mean of Bonamente. et al. (2006) data. ${ }^{(c)}$ Electron temperature of the cluster obtained by fitting the isothermal beta-model to X-ray data (Bonamente et al. 2006).

This distribution is a generalization of a double beta-model profile of the electron number density, developed by La Roque et al. (2005), but instead using the same $\beta$ parameter for both the central region and the outskirts, as in Bonamente et al. (2006). The $r_{\mathrm{c} 1}=\theta_{\mathrm{c} 1} / D_{\mathrm{A}}$ and $r_{\mathrm{c} 2}=\theta_{\mathrm{c} 2} / D_{\mathrm{A}}$ are the core radii of the inner and outer distributions, and $f$ is a parameter defined between 0 and 1 that represents how the core region dominates the outer region. These parameters, together with $n_{\mathrm{e} 0}$, are taken from Bonamente et al. (2006) and summarized in Table 1 for the selected clusters.

We describe the temperature profile as

$\frac{T_{\mathrm{e}, \mathrm{CC}}(r)}{T_{\mathrm{X}}}= \begin{cases}A_{1}\left(\frac{r}{r_{500}}\right)^{m_{1}} & \text { for } \frac{r}{r_{500}}<0.1 \\ A_{2}\left(\frac{r}{r_{500}}\right)^{m_{2}} & \text { for } \frac{r}{r_{500}}>0.2\end{cases}$

where $A_{1,2}$ are determined by fixing the position at which the two power laws, described by the $m_{1}$ and $m_{2}$ parameters, intersect each other, as explained in Appendix A.

The search for a universal temperature profile in the cluster halo region has been a target of several works based on observations (e.g. Markevitch et al. 1998; De Grandi \& Molendi 2002; Zhang et al. 2004; Vikhlinin et al. 2005, 2006; Sanderson et al. 2006; Zhang et al. 2007; Pratt et al. 2007; Zhang et al. 2008) and hydrodynamical simulations (e.g., Loken et al. 2002; Borgani et al. 2004; Kay et al. 2004; Piffaretti \& Valdarnini 2008).

The profile, proposed in this paper specifically for CC clusters, follows both the central drop and the outer decline of the gas temperature. The function is formalized in the $\log \left(r / r_{500}\right)-$ $\log \left(T_{\mathrm{e}}(r) / T_{\mathrm{X}}\right)$ plane on which the power laws of Eq. (8) become linear functions (see Appendix A for a complete treatment). To fix the parameters $A_{1}$ and $A_{2}$ of the radial electron temperature profile and to confirm the power laws indices $m_{1}$ and $m_{2}$, we fit a co-adding of Chandra electron temperature normalized to $T_{\mathrm{X}}$ data, of the CC clusters selection with the proposed function. $T_{\mathrm{X}}$ represents the average temperature value in the range

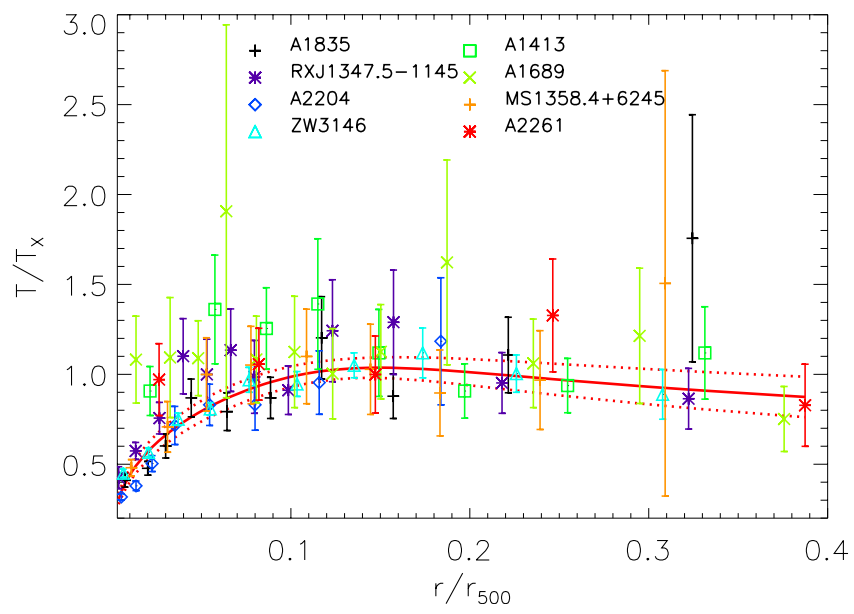

Fig. 1. Radial electron temperature profiles of a Chandra-selected sample of CC galaxy clusters (Bonamente et al. 2006) and the best fit (solid line) of the electron temperature profile with the $1 \sigma$ error (dotted lines) proposed in this work. Temperatures and radii are expressed in terms of $T_{\mathrm{X}}$ and $r_{500}$, respectively, which are used as scale quantities throughout this work.

$(0.1 \div 1.0) r_{500}$, and it is used to scale each cluster, in order to fit the universal temperature function to the measured profiles. In Table 2 we report the cluster redshift, $z$, and the angular diameter distance, $D_{\mathrm{A}}$. The scale radius $r_{500}$ and temperature $T_{\mathrm{X}}$ are also collected. The chosen radial range, which is used to calculate the scale temperature $T_{\mathrm{X}}$, corresponds to a cut in the central region $\left(r<0.1 r_{500}\right)$. Obviously this value cannot be compared easily with results of other works because it strictly depends on the data radial extension. In fact, an important source of bias is the temperature definition (Vikhlinin et al. 2006). Here, we use the spectroscopic temperature $T_{\mathrm{X}}$. In Fig. 1 the temperature data of our cluster sample with the best fit are plotted. By 
Table 3. Parameters of the single beta-model, estimated using the MCMC procedure described in the text, as best fit of the CC cluster maps.

\begin{tabular}{llccc}
\hline \hline Cluster & $\begin{array}{l}F W H M \text { fov } \\
(\operatorname{arcmin})\end{array}$ & $\begin{array}{c}\Delta T_{\text {SZ0 }} \\
(\mu \mathrm{K})\end{array}$ & $\beta$ & $\begin{array}{c}\theta_{\mathrm{c}} \\
(\operatorname{arcsec})\end{array}$ \\
\hline $\mathrm{Cl}_{\mathrm{A} 1413}$ & 1 & $-273 \pm 9$ & $0.731 \pm 0.006$ & $66.1 \pm 3.0$ \\
& 4.5 & $-224 \pm 14$ & $0.743 \pm 0.009$ & $81.7 \pm 7.3$ \\
$\mathrm{Cl}_{\mathrm{A} 1689}$ & 7 & $-195 \pm 11$ & $0.758 \pm 0.011$ & $96.4 \pm 8.0$ \\
& 1 & $-554 \pm 8$ & $0.979 \pm 0.006$ & $80.2 \pm 1.5$ \\
& 4.5 & $-430 \pm 12$ & $0.994 \pm 0.004$ & $93.7 \pm 2.2$ \\
$\mathrm{Cl}_{\mathrm{A} 1835}$ & 7 & $-592 \pm 84$ & $0.909 \pm 0.018$ & $61.4 \pm 8.3$ \\
& 1 & $-709 \pm 10$ & $0.951 \pm 0.006$ & $56.8 \pm 1.1$ \\
& 4.5 & $-468 \pm 29$ & $0.980 \pm 0.013$ & $76.0 \pm 5.0$ \\
$\mathrm{Cl}_{\mathrm{A} 2204}$ & 7 & $-818 \pm 88$ & $0.870 \pm 0.010$ & $36.0 \pm 3.6$ \\
& 1 & $-727 \pm 8$ & $0.848 \pm 0.003$ & $68.4 \pm 0.9$ \\
& 4.5 & $-551 \pm 18$ & $0.865 \pm 0.007$ & $86.0 \pm 3.6$ \\
$\mathrm{Cl}_{\mathrm{A} 2261}$ & 7 & $-514 \pm 39$ & $0.859 \pm 0.017$ & $86.9 \pm 9.0$ \\
& 1 & $-399 \pm 10$ & $0.830 \pm 0.006$ & $52.3 \pm 1.7$ \\
$\mathrm{Cl}_{\mathrm{MS} 1358.4+6245}$ & 1 & $-273 \pm 17$ & $0.849 \pm 0.008$ & $71.6 \pm 4.6$ \\
& 4.5 & $-323 \pm 32$ & $0.816 \pm 0.013$ & $55.5 \pm 6.4$ \\
$\mathrm{Cl}_{\mathrm{RXJ1347.5-1145}}$ & 1 & $-304 \pm 12$ & $0.857 \pm 0.011$ & $49.6 \pm 2.7$ \\
& 4.5 & $-245 \pm 28$ & $0.856 \pm 0.013$ & $56.0 \pm 6.1$ \\
& 4.5 & $-1246 \pm 116$ & $0.753 \pm 0.009$ & $8.7 \pm 1.2$ \\
$\mathrm{Cl}_{\mathrm{ZW} 3146}$ & 7 & $-1683 \pm 15$ & $0.835 \pm 0.002$ & $37.7 \pm 0.5$ \\
& 1 & $-893 \pm 30$ & $0.860 \pm 0.005$ & $62.7 \pm 2.5$ \\
& 4.5 & $-739 \pm 41$ & $0.873 \pm 0.011$ & $73.1 \pm 5.1$ \\
& 7 & $-645 \pm 13$ & $0.839 \pm 0.005$ & $42.4 \pm 1.1$ \\
& & $-404 \pm 38$ & $0.857 \pm 0.013$ & $61.3 \pm 6.7$ \\
& & $-585 \pm 77$ & $0.801 \pm 0.011$ & $36.5 \pm 5.2$ \\
\hline
\end{tabular}

following Appendix A, the resulting $T_{\mathrm{e}}$ profile parameters are $A_{1}=2.41 \pm 0.14, A_{2}=0.55 \pm 0.10, m_{1}=0.38 \pm 0.02$ and $m_{2}=-0.29 \pm 0.11$, which univocally define the $T_{\mathrm{e}}(r)$ function. We note that, even if the power law that describes the outskirts of the cluster temperature distribution suffers larger uncertainties, $m_{1}$ and $m_{2}$ are both compatible, within one standard deviation, with estimates available in the literature. For example, Zhang et al. (2008) find $m_{1}=0.38 \pm 0.04$, for $r<0.2 r_{500}$, in agreement with Sanderson et al. (2006), who proposes $m_{1}=0.4$, for $r<0.1 r_{500}$. For radii larger then $0.2 r_{500}$, Zhang et al. (2008) fitted a selected sample of data from XMM-Newton finding structurally similar behavior to ours with $m_{2}=-0.28 \pm 0.19$.

\section{Pipeline of cluster simulation}

The analysis reported in this paper can be summarized in the following steps:

- construction of an SZ signal distortion profile $\Delta T_{\mathrm{SZ}}(\theta)$, using the ICM information coming from existing X-ray observations;

- convolution of the cluster SZ map with several instrumental beam profiles;

- extraction of the ICM parameters as in the assumptions of the isothermal beta-model;

- estimation of the cluster total mass $M_{\mathrm{tot}}$;

- calculation of the bias on cluster total mass due to the incorrect description of the ICM.

In the first step, we generate angular profiles of the SZ signal distortion $\Delta T_{\mathrm{SZ}}(\theta)$, assuming an observing frequency of $150 \mathrm{GHz}$. The electron number density $n_{\mathrm{e}}$ and temperature $T_{\mathrm{e}}$ profiles are constructed using the equations presented in Sect. 2, which we assume to be a good representation of a cool core ICM. The angular profile of the comptonization parameter is then evaluated by projecting the electron pressure profile on the plane orthogonal to the cluster line of sight. The SZ signal is obtained using Eq. (1).

The $\Delta T_{\mathrm{SZ}}(\theta)$ profiles are then convolved by considering three different instrumental beam profiles modeled as a Gaussian, corresponding to large single dishes (SPT or ACT) with $F W H M=$ 1 arcmin, medium size telescopes (MITO or OLIMPO) with $F W H M=4.5$ arcmin, and small apertures (Planck) with $F W H M=7$ arcmin.

The errors associated to the convolved $\Delta T_{\mathrm{SZ}}(\theta)$ profiles are treated as only due to instrumental noise. An optimistic choice of the sensitivity, for all the observatories, is $6 \mu \mathrm{K} / \mathrm{beam}$, corresponding to the Planck channel at $143 \mathrm{GHz}$ (Planck Collaboration 2011c) assuming the necessary integration time on source for the other experiments. Contaminants are not included in the study since we wish to assess our ability to extract the mass of the clusters under ideal conditions.

To simulate the missing knowledge of X-ray observational results, we ignore cluster morphology and assume the most general model for it: an isothermal beta-model, that expressed in temperature is

$\Delta T_{\mathrm{SZ}}=\Delta T_{\mathrm{SZ} 0}\left(1+\frac{\theta^{2}}{\theta_{\mathrm{c}}^{2}}\right)^{\frac{1}{2}-\frac{3}{2} \beta}$.

We apply the Metropolis Hastings (M-H) algorithm Monte Carlo Markov chain (MCMC) procedure to fit this equation (after a convolution with the corresponding instrumental beam) on the simulated profiles to extract the parameters $\Delta T_{\mathrm{SZ} 0}, \beta$ and $\theta_{\mathrm{c}}$. For each cluster, at a fixed field of view (fov), we analyze the accepted set of parameters derived by the MCMC procedure. The degeneracy among the extracted beta-model parameters affects their uncertainties.

We obtain the beta-model parameter set that is most consistent with the $\Delta T_{\mathrm{SZ}}(\theta)$ profile, given the assumed instrumental noise and beam sizes. All the parameters resulting from the MCMC analysis are collected in Table 3 for each cluster and for 
A. Conte et al.: Biased total mass of CC galaxy clusters by SZE measurements

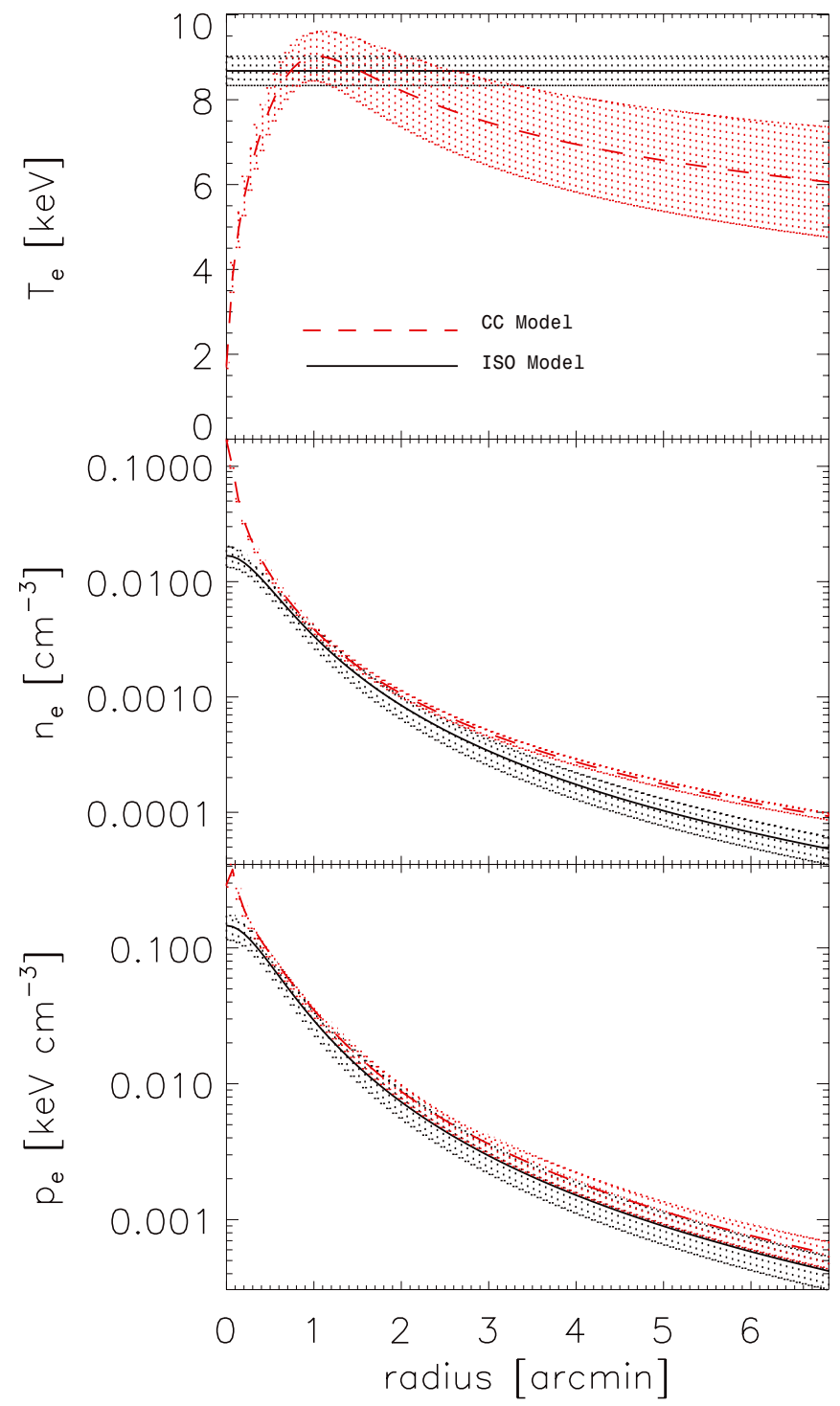

Fig. 2. Radial profiles of the electron temperature, $T_{\mathrm{e}}$ (top), number density, $n_{\mathrm{e}}$ (middle) and pressure, $P_{\mathrm{e}}$ (bottom) for the cluster ZW3146. The red dashed curve describes the CC template while the black solid curve represents the ISO model (the shadowed regions define $1 \sigma$ uncertainties), with parameters extracted by MCMC analysis considering $\Delta T_{\mathrm{SZ}}(\theta)$ profiles convolved with a beam of $7 \operatorname{arcmin}(F W H M)$.

each fov. Figure 2 shows the electron temperature (top), number density (middle), and pressure (bottom) profiles for only the cluster ZW3146, as an example, of both the original CC ICM and the recovered ISO model. The errors associated to the curves account for the $1 \sigma$ uncertainties on the parameters.

\section{Cluster total mass estimation}

We want to stress the consequences of the assumptions on the ICM physics when we miss X-ray information. A quantity, such as the total mass $M_{\text {tot }}$, can be biased by a different physical state of the ICM (i.e. mergers or cooling flow mechanisms). In particular we estimate the mass for both the ICM discussed templates (CC and ISO), by using the following different approaches:

- hydrostatic equilibrium assumption for the cluster gas (hydrostatic equilibrium, HE);

- gas fraction independence of cluster physical state (fixed gas fraction, FGF);
- $M_{\text {tot }}-Y$ scaling relation (scaling law, SL), as derived in the standard self-similar collapse scenario.

The masses are calculated, in particular, within a fixed integration radius $r_{\text {int }}$ (aperture radius), which we arbitrarily choose equal to the $r_{500}$ values as reported in Morandi et al. (2007, see Table 2). This choice is motivated by the need to fix an aperture radius within which to estimate integrated quantities. We point out that $r_{\text {int }}$ does not always correspond to the same overdensity, due to the different assumed ICM templates. It is clear that, hereafter, results associated to the clusters simulated in this work cannot be considered as describing the true ICM physics of the observed objects. We choose, however, to maintain the link with the "native" cluster in the name (NAME $\rightarrow \mathrm{Cl}_{\mathrm{NAME}}$ ).

\subsection{Hydrostatic equilibrium}

The first approach, HE, assumes a spherical symmetry for the cluster, so that the hydrostatic equilibrium equation can be written (Sarazin 1988) as

$\frac{\mathrm{d} P_{\text {gas }}(r)}{\mathrm{d} r}=-\rho_{\text {gas }}(r) G \frac{M_{\text {tot }}(<r)}{r^{2}}$

where $M_{\text {tot }}(<r)$ is the total cluster mass within radius $r$ and under the ideal gas assumption, $P_{\text {gas }}=\left(\rho_{\text {gas }} k_{\mathrm{B}} T_{\text {gas }}\right) /\left(\mu m_{\mathrm{p}}\right)$, with $\rho_{\text {gas }}=\mu_{\mathrm{e}} m_{\mathrm{p}} n_{\mathrm{e}}, \mu$ and $\mu_{\mathrm{e}}$ are the total and electron mean molecular weights (i.e. the mean particle mass per electron in units of the proton mass $m_{\mathrm{p}}$ ), $G$ is the gravitational constant and $T_{\mathrm{gas}}=T_{i}=T_{\mathrm{e}}$, the ion and electron temperatures respectively, because the system is assumed to be in thermal equilibrium. To adapt this equation to the SZ physics, we substitute the gas pressure, which accounts for both electrons and ions, with the simple electron pressure $P_{\mathrm{e}}$, which causes the SZ effect, so we have

$\frac{\mathrm{d} P_{\mathrm{e}}(r)}{\mathrm{d} r}=-\mu m_{\mathrm{p}} G \frac{M_{\mathrm{tot}}(<r)}{r^{2}} n_{\mathrm{e}}(r)$.

The total mass can be derived as

$M_{\mathrm{tot}}(<r)=-\frac{k T_{\mathrm{e}}(r)}{G \mu m_{\mathrm{p}}} r\left[\frac{\partial \ln \left(n_{\mathrm{e}}(r)\right)}{\partial \ln (r)}+\frac{\partial \ln \left(k T_{\mathrm{e}}(r)\right)}{\partial \ln (r)}\right]$,

which reduces, in the simple case of the isothermal beta-model, to

$M_{\mathrm{tot}}(<r)=\frac{3 \beta k T_{\mathrm{e}}}{G \mu m_{\mathrm{p}}} \frac{r^{3}}{r_{\mathrm{c}}^{2}+r^{2}}$

The previous equations yield $M_{\mathrm{tot}, \mathrm{HE}, \mathrm{CC}}$ and $M_{\mathrm{tot}, \mathrm{HE}, \mathrm{ISO}}$, respectively. If we also calculate the cluster gas mass by

$M_{\text {gas }}=\mu_{\mathrm{e}} m_{\mathrm{p}} \int n_{\mathrm{e}} \mathrm{d} V$

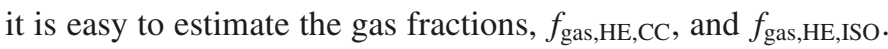
The plots in Fig. 3 refer, still as an example, to the cluster $\mathrm{Cl}_{\text {ZW3146 }}$ and show the radial profiles of the cumulative cluster total and gas masses for both the ICM templates and the gas fraction. The $1 \sigma$ lines are derived by considering the uncertainties of the ICM parameters. 


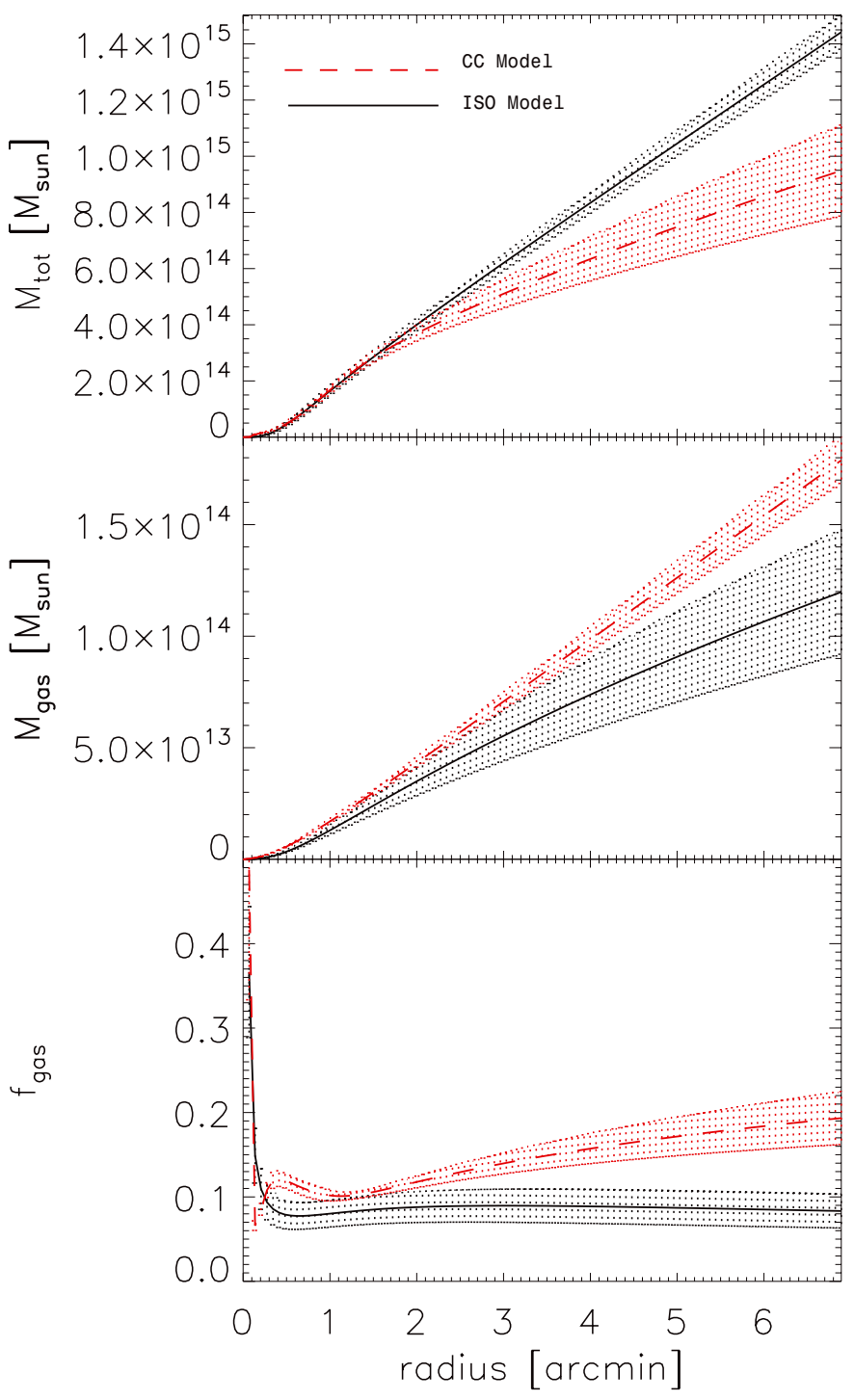

Fig. 3. Radial profiles of the cumulative total (top) and gas (middle) cluster masses and the gas fraction (bottom) for the cluster $\mathrm{Cl}_{\mathrm{ZW} 3146}$. The plots refer to the two ICM templates (CC and ISO) discussed in the text, under the hydrostatic hypothesis. The ISO model parameters have been extracted by MCMC analysis considering $\Delta T_{\mathrm{SZ}}(\theta)$ profiles convolved with a beam of $7 \operatorname{arcmin}(F W H M)$.

\subsection{Fixed gas fraction}

The second approach, FGF, assumes that the gas fraction, estimated at $r_{\text {int }}$, is independent of the cluster dynamical state, as deduced by simulations (e.g. Rasia et al. 2006; Lau et al. 2009) and as recently derived by XMM-Newton and Subaru observations (Zhang et al. 2010). Under this hypothesis, we can derive the cluster total masses directly by gas masses as $M_{\mathrm{tot}, \mathrm{FGF}}=$ $M_{\text {gas }} / f_{\text {gas }}$.

Because the integrated quantities are calculated by referring to a fixed radius $r_{\text {int }}$, defined in the previous section, it is evident that, for each cluster and for each considered ICM model, we are dealing with different overdensities. We calculate these overdensities by

$\Delta_{\text {int }}=\frac{2 G}{H_{0}^{2} E(z)^{2} r_{\text {int }}^{3}} M_{\text {tot }}\left(r_{\text {int }}\right)$.

The results obtained for the HE and FGF approaches are collected in Table 4. In order to derive all the quantities corresponding to the ISO template from the parameters obtained with the MCMC procedure, we need to convert the $\Delta T_{\mathrm{Sz} 0}$ values to central electron number densities. This is possible using equation

$n_{\mathrm{e} 0}=\frac{\Delta T_{\mathrm{SZ} 0}}{T_{\mathrm{CMB}} f(x)} \frac{m_{\mathrm{e}} c^{2}}{\sigma_{\mathrm{T}} \theta_{\mathrm{c}} D_{\mathrm{A}} \sqrt{\pi}} \frac{\Gamma\left(\frac{3}{2} \beta\right)}{\Gamma\left(\frac{3}{2} \beta-\frac{1}{2}\right)} \frac{1}{T_{\mathrm{e} 0}}$,

which is derived by expressing Eq. (5) in terms of $\Delta T_{\mathrm{SZ}}$. Of course this expression introduces a degeneracy between temperature and density, possibly producing the same $\Delta T_{\mathrm{SZ} 0}$ value. For this reason the masses presented in this work have been obtained by fixing the electron temperature to the $T_{\mathrm{X}}$ values (Table 2 ) and assuming $f_{\text {gas }}=0.1$.

\section{3. $M_{\mathrm{tot}}-Y$ scaling relation}

The total cluster mass can be inferred by applying a self-similar relation that links it to the integrated comptonization parameter. The $M_{\mathrm{tot}}-Y$ scaling law is usually calibrated for a fixed overdensity value, while in this work we are dealing with masses calculated at different overdensities. For this reason in the scaling law, we make the dependence on overdensity explicit. Simple considerations, based on the assumption that cluster evolution is completely determined by gravitational processes, lead to an easy scaling relation that connects the total mass of a cluster of galaxies, $M_{\text {tot }}$, to its temperature, $T_{\mathrm{e}}$, considering an isothermal structure for the ICM. Following Kravtsov et al. (2006) and Bryan $\&$ Norman (1998) and keeping the overdensity dependence explicit, we have

$k_{\mathrm{B}} T_{\mathrm{e}}=\mu m_{\mathrm{p}}\left[\frac{27}{16} \Delta_{\mathrm{int}} G^{2} H_{0}^{2} E(z)^{2}\right]^{1 / 3} M_{\mathrm{tot}}^{2 / 3}$.

To connect a spherically integrated quantity (e.g. cluster total mass) to one derived by a cylindrical integration (e.g. integrated SZ flux), we consider the spherical analogous to the SZ flux, $Y_{\mathrm{S}}$. This quantity is directly proportional to the cluster total mass as

$Y_{\mathrm{S}}=\frac{k_{\mathrm{B}} \sigma_{\mathrm{T}}}{m_{\mathrm{e}} c^{2}} \int n_{\mathrm{e}} T_{\mathrm{e}} \mathrm{d} V=\frac{k_{\mathrm{B}} \sigma_{\mathrm{T}}}{m_{\mathrm{e}} c^{2}} T_{\mathrm{mw}} \frac{f_{\text {gas }} M_{\mathrm{tot}}}{\mu_{\mathrm{e}} m_{\mathrm{p}}}$

where $M_{\mathrm{tot}}$ is the cluster total mass inside a sphere with radius equal to $r_{\mathrm{int}}$, and $T_{\mathrm{mw}}$ is the gas mass-weighted mean temperature.

By combining the previous equations, we get

$M_{\text {tot }}=0.248\left[f_{\text {gas }}^{3} E(z)^{2} \Delta_{\text {int }}\right]^{-1 / 5} Y_{\mathrm{S}}^{3 / 5} 10^{14} M_{\odot}$.

This means that, by estimating $Y_{\mathrm{S}}$, we can easily infer the corresponding total cluster mass. Furthermore, in order to derive the integrated SZ flux, we need to solve the equation

$Y=2 \pi \int_{0}^{\theta_{\mathrm{int}}} y(\theta) \theta \mathrm{d} \theta$,

where $\theta_{\text {int }}=r_{\text {int }} / D_{\mathrm{A}}$ and $y(\theta)$ is extracted by the simulated SZ temperature decrement profiles (in the $\mathrm{CC}$ case) or calculated directly from Eq. (4), by considering the parameters as derived from the MCMC analysis in Sect. 3 (in the ISO case). The two defined integrated Comptonization parameters $\left(Y_{\mathrm{S}}\right.$ and $\left.Y\right)$ are connected by the dimensionless quantity $C=D_{\mathrm{A}}^{2} Y / Y_{\mathrm{S}}$. 
Table 4. Total cluster masses calculated considering the HE and FGF approaches.

\begin{tabular}{|c|c|c|c|}
\hline Cluster & $\begin{array}{l}\text { ICM template } \\
\text { ( } F W H M \text { fov) }\end{array}$ & $\begin{array}{c}M_{\mathrm{tot}, \mathrm{HE}} \\
\left(10^{14} M_{\odot}\right)\end{array}$ & $\begin{array}{l}M_{\mathrm{tot}, \mathrm{FGF}}{ }^{a} \\
\left(10^{14} M_{\odot}\right)\end{array}$ \\
\hline A1413 & $\mathrm{CC}$ & $3.85 \pm 0.77$ & $8.73 \pm 2.03$ \\
\hline \multirow[t]{3}{*}{$\mathrm{Cl}_{\mathrm{A} 1413}$} & ISO $\left(1^{\prime}\right)$ & $6.59 \pm 0.60$ & $6.21 \pm 0.35$ \\
\hline & ISO $\left(4.5^{\prime}\right)$ & $6.67 \pm 0.59$ & $6.02 \pm 0.59$ \\
\hline & ISO $\left(7^{\prime}\right)$ & $6.66 \pm 0.58$ & $5.93 \pm 0.89$ \\
\hline A1689 & $\mathrm{CC}$ & $8.96 \pm 1.97$ & $12.72 \pm 2.33$ \\
\hline \multirow[t]{3}{*}{$\mathrm{Cl}_{\mathrm{A} 1689}$} & ISO (1') & $13.56 \pm 1.41$ & $9.88 \pm 3.12$ \\
\hline & ISO $\left(4.5^{\prime}\right)$ & $13.61 \pm 1.33$ & $9.14 \pm 4.08$ \\
\hline & ISO $\left(7^{\prime}\right)$ & $12.66 \pm 1.33$ & $8.86 \pm 2.01$ \\
\hline A1835 & $\mathrm{CC}$ & $10.23 \pm 2.30$ & $12.72 \pm 1.18$ \\
\hline \multirow[t]{3}{*}{$\mathrm{Cl}_{\mathrm{A} 1835}$} & ISO $\left(1^{\prime}\right)$ & $16.21 \pm 1.61$ & $10.08 \pm 0.33$ \\
\hline & $\operatorname{ISO}\left(4.5^{\prime}\right)$ & $16.32 \pm 1.55$ & $9.13 \pm 0.94$ \\
\hline & ISO $\left(7^{\prime}\right)$ & $15.08 \pm 1.40$ & $7.99 \pm 1.44$ \\
\hline A2204 & $\mathrm{CC}$ & $12.91 \pm 3.30$ & $14.84 \pm 2.96$ \\
\hline \multirow[t]{3}{*}{$\mathrm{Cl}_{\mathrm{A} 2204}$} & ISO $\left(1^{\prime}\right)$ & $19.88 \pm 3.27$ & $10.13 \pm 0.24$ \\
\hline & ISO $\left(4.5^{\prime}\right)$ & $20.33 \pm 3.05$ & $10.87 \pm 0.67$ \\
\hline & ISO $\left(7^{\prime}\right)$ & $19.89 \pm 2.86$ & $10.32 \pm 1.54$ \\
\hline A2261 & $\mathrm{CC}$ & $4.79 \pm 1.11$ & $10.55 \pm 3.48$ \\
\hline \multirow[t]{3}{*}{$\mathrm{Cl}_{\mathrm{A} 2261}$} & ISO $\left(1^{\prime}\right)$ & $7.88 \pm 1.09$ & $7.80 \pm 3.50$ \\
\hline & ISO $\left(4.5^{\prime}\right)$ & $7.84 \pm 1.18$ & $7.24 \pm 1.22$ \\
\hline & ISO (7') & $7.81 \pm 1.08$ & $6.94 \pm 1.09$ \\
\hline MS1358.4+6245 & $\mathrm{CC}$ & $8.09 \pm 1.75$ & $10.23 \pm 1.65$ \\
\hline \multirow[t]{3}{*}{$\mathrm{Cl}_{\mathrm{MS} 1358.4+6245}$} & ISO $\left(1^{\prime}\right)$ & $13.27 \pm 1.77$ & $7.74 \pm 0.63$ \\
\hline & ISO $\left(4.5^{\prime}\right)$ & $13.17 \pm 1.88$ & $7.27 \pm 1.22$ \\
\hline & ISO $\left(7^{\prime}\right)$ & $11.90 \pm 1.51$ & $6.12 \pm 1.14$ \\
\hline RXJ1347.5-1145 & $\mathrm{CC}$ & $14.66 \pm 3.02$ & $32.34 \pm 4.05$ \\
\hline \multirow[t]{3}{*}{$\mathrm{Cl}_{\mathrm{RXJ1347.5-1145}}$} & ISO (1') & $24.45 \pm 2.49$ & $25.04 \pm 0.48$ \\
\hline & ISO $\left(4.5^{\prime}\right)$ & $24.47 \pm 2.54$ & $22.08 \pm 1.30$ \\
\hline & ISO $\left(7^{\prime}\right)$ & $24.66 \pm 2.49$ & $21.07 \pm 1.84$ \\
\hline & $\mathrm{CC}$ & $9.06 \pm 1.64$ & $17.88 \pm 1.05$ \\
\hline \multirow{3}{*}{$\mathrm{Cl}_{\mathrm{ZW} 3146}$} & ISO $\left(1^{\prime}\right)$ & $15.05 \pm 0.60$ & $13.77 \pm 0.58$ \\
\hline & ISO $\left(4.5^{\prime}\right)$ & $15.14 \pm 0.67$ & $12.72 \pm 1.97$ \\
\hline & ISO $\left(7^{\prime}\right)$ & $14.44 \pm 0.64$ & $12.03 \pm 2.61$ \\
\hline
\end{tabular}

Notes. ${ }^{(a)}$ Derived by $M_{\text {gas }}$ assuming $f_{\text {gas }}=0.1$.

We averaged the $C$ factors, assuming different cylindric depths equal to 2,5 , and $10 r_{\text {int }}$, obtaining $C_{2}=1.52 \pm 0.07$, $C_{5}=1.85 \pm 0.14$, and $C_{10}=2.00 \pm 0.19$, respectively, converging to the value in Bonamente et al. (2008).

The scaling law can be rewritten as

$M_{\text {tot }}=0.248\left[f_{\text {gas }}^{3} E(z)^{2} C_{\infty}^{3} \Delta_{\text {int }}\right]^{-1 / 5}\left(D_{\mathrm{A}}^{2} Y\right)^{3 / 5} 10^{14} M_{\odot}$,

which can directly give an estimation of cluster total mass

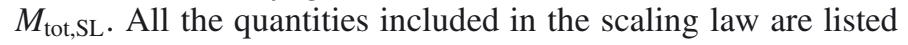
in Table 5.

\section{Bias on the total mass}

The results clearly show that a general interpretation of cluster physics, as assumed in a pure self-similar scaling law like $M_{\text {tot }}-$ $Y$, can infer a wrong estimate of cluster total mass. To emphasize and to quantify this point, we define a mass bias as

$M B=\frac{M_{\mathrm{tot}, \mathrm{ISO}}-M_{\mathrm{tot}, \mathrm{CC}}}{M_{\mathrm{tot}, \mathrm{CC}}}$.

This quantifies the difference between the SZ derived mass, as results from the isothermal beta-model assumption (ISO) and the $\mathrm{X}$-ray derived mass (CC), which we deduce from the HE, FGF, and SL approaches. We notice no significant bias dependence on the beam size used to convolve the SZ signals, under the assumption of the presence of instrumental noise alone. Therefore, all plots refers to results obtained with a fov of $7 \operatorname{arcmin} F W H M$, considering $T_{\mathrm{X}}$ values as in Table 2 . We calculate the mass bias for all the analyzed approaches.

Since the $\Delta T_{\text {SZ0 }}$ parameter does not give us unique information on the physics of the ICM, we have to study different pairs of the parameters $n_{\mathrm{e} 0}$ and $T_{\mathrm{x}}$. Thus, we select values of $T_{\mathrm{x}}$ that describe a reliable range of electron temperatures (from $5 \mathrm{keV}$ to $15 \mathrm{keV}$ ) and calculate the corresponding $n_{\mathrm{e} 0}$. The mass biases are plotted in Fig. 4a, where the three cases (HE, FGF, and $\mathrm{SL}$ ) are shown all together in the plots corresponding to clusters $\mathrm{Cl}_{\mathrm{A} 1689}, \mathrm{Cl}_{\mathrm{A} 2204}$, and $\mathrm{Cl}_{\mathrm{RXJ1347.5-1145}}$, representing a wide span in the electron temperature values. Table 6 lists the mass biases, as estimated at the $\mathrm{X}$-ray derived electron temperature $\left(T_{\mathrm{X}}\right.$ in Table 2).

A check of the goodness of the procedure was done by simulating the observation of a cluster having an isothermal betamodel $\Delta T_{\mathrm{SZ}}(\theta)$ profile instead of a CC cluster. For this validation procedure, named Test, we used the same analytical expression of the SZ signal to extract the parameters. The assumed electron temperature is the one obtained by Bonamente et al. (2006) and reported in the last column in Table 2. In Fig. 4b we represent the cluster total mass bias with these assumptions. While the bias is always zero in the HE approach, for the FGF and SL cases, as expected, we notice a mass bias dependence on $T_{\mathrm{e}}$. Due to the degeneracy between the electron temperature and number density, the mass bias varies with $n_{\mathrm{e}}$. In all cases, it is worth noting that these biases nullify for electron temperatures equal 
A\&A 532, A14 (2011)

Table 5. Total mass as derived by applying the $M_{\mathrm{tot}}-Y$ scaling law as in Eq. (21).

\begin{tabular}{|c|c|c|c|c|c|}
\hline $\begin{array}{l}\text { Cluster } \\
\text { name }\end{array}$ & $\begin{array}{l}\text { ICM template } \\
\text { ( } F W H M \text { fov })\end{array}$ & $\begin{array}{c}D_{\mathrm{A}}^{2} Y \\
\left(\mathrm{kpc}^{2}\right)\end{array}$ & $f_{\mathrm{gas}}$ & $\Delta_{\text {int }}$ & $\begin{array}{c}M_{\mathrm{tot}, \mathrm{SL}} \\
\left(10^{14} M_{\odot}\right)\end{array}$ \\
\hline A1413 & $\mathrm{CC}$ & $100 \pm 1$ & $0.23 \pm 0.05$ & $345 \pm 69$ & $1.94 \pm 0.23$ \\
\hline \multirow[t]{3}{*}{$\mathrm{Cl}_{\mathrm{A} 1413}$} & ISO (1') & $95 \pm 5$ & $0.10 \pm 0.01$ & $591 \pm 53$ & $2.81 \pm 0.18$ \\
\hline & $\operatorname{ISO}\left(4.5^{\prime}\right)$ & $92 \pm 12$ & $0.09 \pm 0.01$ & $598 \pm 53$ & $2.86 \pm 0.30$ \\
\hline & ISO (7') & $90 \pm 9$ & $0.09 \pm 0.01$ & $597 \pm 52$ & $2.81 \pm 0.26$ \\
\hline A1689 & $\mathrm{CC}$ & $177 \pm 2$ & $0.15 \pm 0.03$ & $477 \pm 105$ & $3.34 \pm 0.38$ \\
\hline \multirow{3}{*}{$\mathrm{Cl}_{\mathrm{A} 1689}$} & ISO (1') & $152 \pm 6$ & $0.07 \pm 0.01$ & $722 \pm 75$ & $4.15 \pm 0.23$ \\
\hline & ISO $\left(4.5^{\prime}\right)$ & $142 \pm 6$ & $0.07 \pm 0.01$ & $724 \pm 71$ & $4.17 \pm 0.23$ \\
\hline & ISO $\left(7^{\prime}\right)$ & $139 \pm 32$ & $0.07 \pm 0.02$ & $674 \pm 71$ & $4.16 \pm 0.82$ \\
\hline A1835 & $\mathrm{CC}$ & $263 \pm 2$ & $0.13 \pm 0.03$ & $468 \pm 105$ & $4.48 \pm 0.36$ \\
\hline \multirow{3}{*}{$\mathrm{Cl}_{\mathrm{A} 1835}$} & ISO $\left(1^{\prime}\right)$ & $186 \pm 7$ & $0.06 \pm 0.01$ & $741 \pm 74$ & $5.05 \pm 0.25$ \\
\hline & ISO $\left(4.5^{\prime}\right)$ & $171 \pm 17$ & $0.06 \pm 0.01$ & $746 \pm 71$ & $5.12 \pm 0.51$ \\
\hline & ISO $\left(7^{\prime}\right)$ & $152 \pm 28$ & $0.05 \pm 0.01$ & $689 \pm 64$ & $5.00 \pm 0.85$ \\
\hline A2204 & $\mathrm{CC}$ & $271 \pm 2$ & $0.12 \pm 0.04$ & $337 \pm 86$ & $5.28 \pm 0.72$ \\
\hline \multirow[t]{3}{*}{$\mathrm{Cl}_{\mathrm{A} 2204}$} & ISO $\left(1^{\prime}\right)$ & $241 \pm 6$ & $0.06 \pm 0.01$ & $520 \pm 86$ & $6.78 \pm 0.47$ \\
\hline & ISO $\left(4.5^{\prime}\right)$ & $231 \pm 15$ & $0.05 \pm 0.01$ & $531 \pm 80$ & $6.89 \pm 0.59$ \\
\hline & ISO $\left(7^{\prime}\right)$ & $222 \pm 31$ & $0.05 \pm 0.01$ & $520 \pm 75$ & $6.88 \pm 0.99$ \\
\hline A2261 & $\mathrm{CC}$ & $134 \pm 2$ & $0.22 \pm 0.06$ & $388 \pm 90$ & $2.31 \pm 0.38$ \\
\hline \multirow[t]{3}{*}{$\mathrm{Cl}_{\mathrm{A} 2261}$} & ISO $\left(1^{\prime}\right)$ & $110 \pm 5$ & $0.10 \pm 0.02$ & $639 \pm 89$ & $2.87 \pm 0.19$ \\
\hline & ISO $\left(4.5^{\prime}\right)$ & $104 \pm 9$ & $0.09 \pm 0.02$ & $635 \pm 95$ & $2.93 \pm 0.29$ \\
\hline & ISO $\left(7^{\prime}\right)$ & $99 \pm 16$ & $0.09 \pm 0.02$ & $633 \pm 88$ & $2.93 \pm 0.48$ \\
\hline MS1358.4+6245 & $\mathrm{CC}$ & $166 \pm 4$ & $0.13 \pm 0.03$ & $233 \pm 50$ & $3.82 \pm 0.35$ \\
\hline \multirow[t]{3}{*}{$\mathrm{Cl}_{\mathrm{MS} 1358.4+6245}$} & ISO (1') & $127 \pm 11$ & $0.06 \pm 0.01$ & $381 \pm 51$ & $4.67 \pm 0.45$ \\
\hline & ISO $\left(4.5^{\prime}\right)$ & $113 \pm 21$ & $0.06 \pm 0.01$ & $379 \pm 54$ & $4.56 \pm 0.76$ \\
\hline & ISO $\left(7^{\prime}\right)$ & $96 \pm 18$ & $0.05 \pm 0.01$ & $342 \pm 43$ & $4.36 \pm 0.76$ \\
\hline RXJ1347.5-1145 & $\mathrm{CC}$ & $1165 \pm 4$ & $0.23 \pm 0.03$ & $305 \pm 63$ & $8.09 \pm 0.52$ \\
\hline \multirow[t]{3}{*}{$\mathrm{Cl}_{\mathrm{RXJ1347.5-1145}}$} & ISO $\left(1^{\prime}\right)$ & $719 \pm 14$ & $0.10 \pm 0.01$ & $509 \pm 52$ & $8.68 \pm 0.40$ \\
\hline & ISO $\left(4.5^{\prime}\right)$ & $654 \pm 41$ & $0.09 \pm 0.01$ & $509 \pm 52$ & $8.86 \pm 0.60$ \\
\hline & ISO $\left(7^{\prime}\right)$ & $649 \pm 66$ & $0.09 \pm 0.01$ & $513 \pm 52$ & $9.11 \pm 0.80$ \\
\hline ZW3146 & $\mathrm{CC}$ & $312 \pm 4$ & $0.20 \pm 0.04$ & $201 \pm 37$ & $4.42 \pm 0.31$ \\
\hline \multirow[t]{3}{*}{$\mathrm{Cl}_{\mathrm{ZW} 3146}$} & ISO $\left(1^{\prime}\right)$ & $224 \pm 11$ & $0.09 \pm 0.01$ & $334 \pm 13$ & $5.21 \pm 0.23$ \\
\hline & ISO $\left(4.5^{\prime}\right)$ & $208 \pm 38$ & $0.08 \pm 0.01$ & $336 \pm 15$ & $5.31 \pm 0.81$ \\
\hline & ISO $\left(7^{\prime}\right)$ & $205 \pm 43$ & $0.08 \pm 0.02$ & $321 \pm 14$ & $5.41 \pm 0.11$ \\
\hline
\end{tabular}

Table 6. Biases on the cluster total mass estimate for three different approaches as derived by three different fovs.

\begin{tabular}{lcccc}
\hline \hline $\begin{array}{l}\text { Cluster } \\
\text { name }\end{array}$ & $\begin{array}{c}F W H M \text { fov } \\
(\mathrm{arcmin})\end{array}$ & $M B_{\mathrm{HE}}$ & $M B_{\mathrm{FGF}}$ & $M B_{\mathrm{SL}}$ \\
\hline $\mathrm{Cl}_{\mathrm{A} 1413}$ & 1 & $0.75 \pm 0.31$ & $-0.29 \pm 0.19$ & $0.58 \pm 0.32$ \\
& 4.5 & $0.79 \pm 0.35$ & $-0.30 \pm 0.15$ & $0.59 \pm 0.34$ \\
$\mathrm{Cl}_{\mathrm{A} 1689}$ & 7 & $0.81 \pm 0.31$ & $-0.33 \pm 0.17$ & $0.61 \pm 0.31$ \\
& 1 & $0.56 \pm 0.31$ & $-0.24 \pm 0.14$ & $0.43 \pm 0.24$ \\
& 4.5 & $0.59 \pm 0.33$ & $-0.30 \pm 0.15$ & $0.45 \pm 0.28$ \\
$\mathrm{Cl}_{\mathrm{A} 1835}$ & 7 & $0.50 \pm 0.30$ & $-0.35 \pm 0.20$ & $0.55 \pm 0.45$ \\
& 1 & $0.63 \pm 0.31$ & $-0.26 \pm 0.07$ & $0.44 \pm 0.16$ \\
& 4.5 & $0.64 \pm 0.28$ & $-0.32 \pm 0.09$ & $0.47 \pm 0.21$ \\
$\mathrm{Cl}_{\mathrm{A} 2204}$ & 7 & $0.51 \pm 0.32$ & $-0.41 \pm 0.11$ & $0.45 \pm 0.29$ \\
& 1 & $0.59 \pm 0.32$ & $-0.25 \pm 0.15$ & $0.45 \pm 0.27$ \\
$\mathrm{Cl}_{\mathrm{A} 2261}$ & 4.5 & $0.62 \pm 0.30$ & $-0.29 \pm 0.15$ & $0.50 \pm 0.27$ \\
& 7 & $0.63 \pm 0.30$ & $-0.31 \pm 0.18$ & $0.47 \pm 0.33$ \\
$\mathrm{Cl}_{\mathrm{MS} 1358.4+6245}$ & 1 & $0.75 \pm 0.30$ & $-0.23 \pm 0.25$ & $0.52 \pm 0.36$ \\
& 4.5 & $0.74 \pm 0.33$ & $-0.28 \pm 0.29$ & $0.53 \pm 0.40$ \\
& 7 & $0.69 \pm 0.32$ & $-0.30 \pm 0.25$ & $0.54 \pm 0.40$ \\
$\mathrm{Cl}_{\mathrm{RXJ} 1347.5-1145}$ & 1 & $0.71 \pm 0.31$ & $-0.28 \pm 0.12$ & $0.50 \pm 0.21$ \\
& 7.5 & $0.69 \pm 0.34$ & $-0.32 \pm 0.17$ & $0.55 \pm 0.34$ \\
& 1 & $0.52 \pm 0.29$ & $-0.43 \pm 0.14$ & $0.47 \pm 0.32$ \\
$\mathrm{Cl}_{\mathrm{ZW} 3146}$ & 7.5 & $0.79 \pm 0.32$ & $-0.26 \pm 0.08$ & $0.51 \pm 0.17$ \\
& 7 & $0.77 \pm 0.30$ & $-0.35 \pm 0.08$ & $0.56 \pm 0.19$ \\
& 1 & $0.66 \pm 0.38$ & $-0.38 \pm 0.10$ & $0.61 \pm 0.26$ \\
& 4.5 & $0.67 \pm 0.32$ & $-0.28 \pm 0.06$ & $0.47 \pm 0.16$ \\
& 7 & $0.62 \pm 0.33$ & $-0.40 \pm 0.12$ & $0.53 \pm 0.27$ \\
& & & & $0.57 \pm 0.31$ \\
\hline
\end{tabular}


(a)

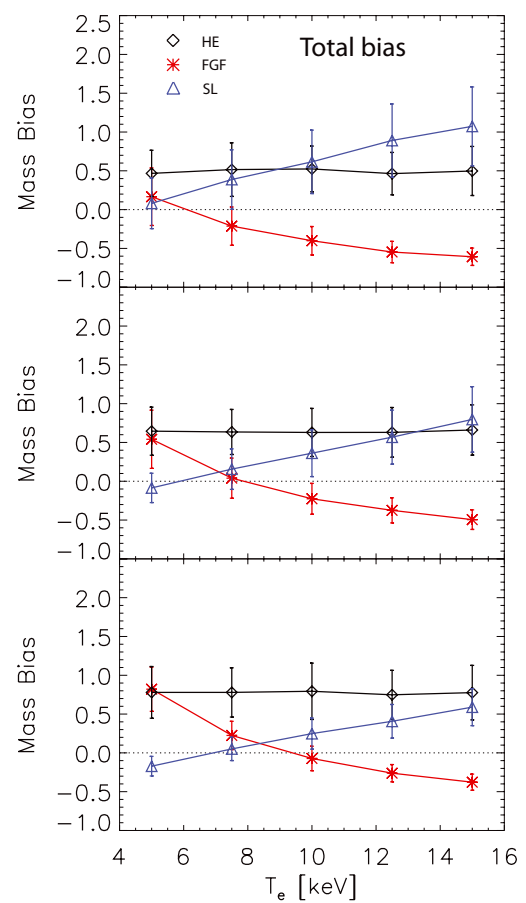

(b)

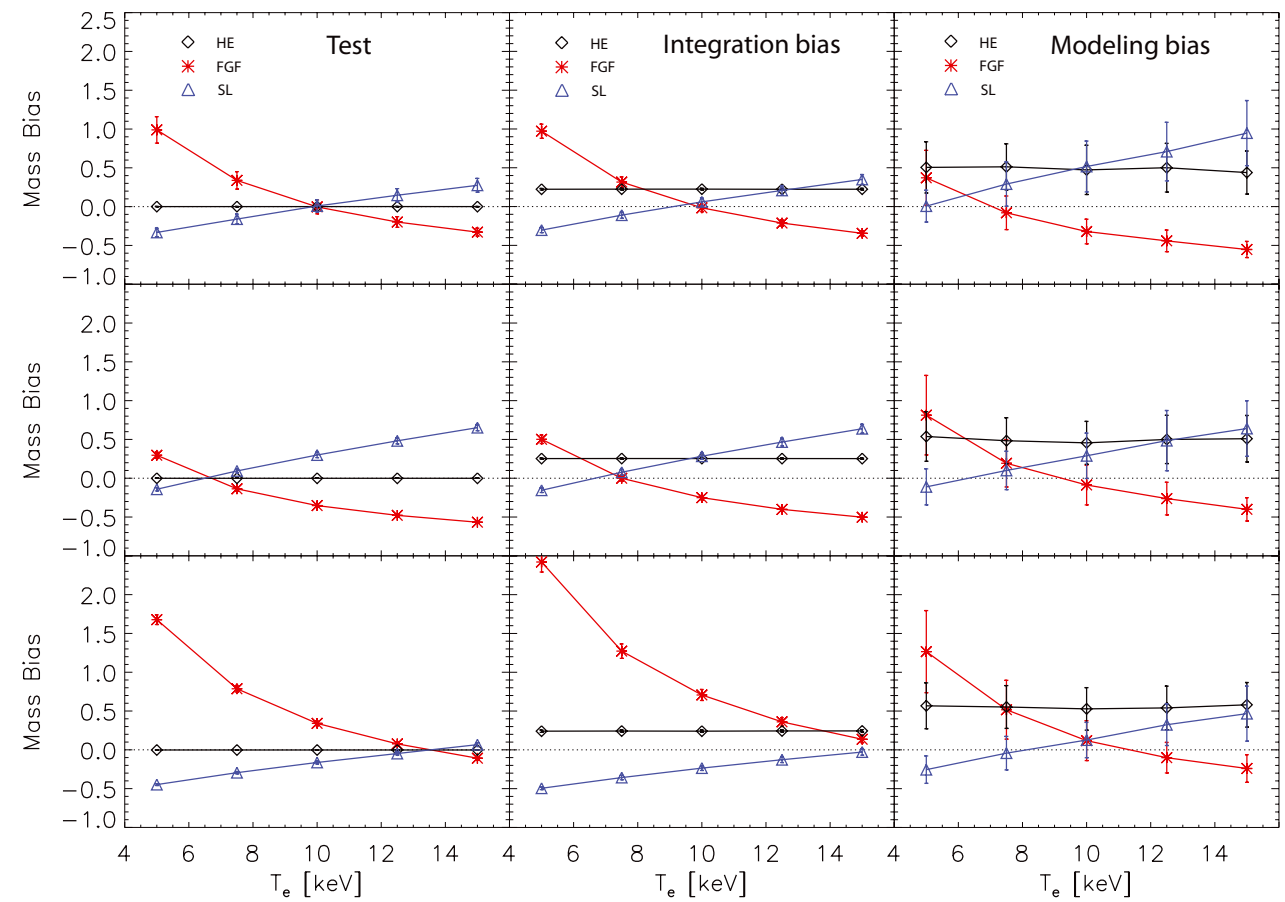

Fig. 4. Total mass biases estimated with different approaches, HE (black), FGF (red), SL (blue), for three representative clusters of our sample (from top to bottom: $\mathrm{Cl}_{\mathrm{A} 1689}, \mathrm{Cl}_{\mathrm{A} 2204}$, and $\mathrm{Cl}_{\mathrm{RXJ1347.5-1145}}$ ). The ISO parameters used for the bias estimation are associated to $\mathrm{SZ}$ signals convolved with a beam of $7 \operatorname{arcmin}(F W H M)$. In panel a) the total mass bias is shown, while in panel b) the reliability of bias estimation has been tested. The two bias contributions are disentangled: integration c) and modeling d) biases. Assuming no prior for $T_{\mathrm{e}}$, the mass biases are plotted versus a reliable range of electron temperatures $(5-15 \mathrm{keV})$.

to $T_{\mathrm{e} 0}$ values, thus proving that the method is not affected by systematics.

The degeneracy between the ICM parameters, resulting from yielding the same SZ signal, produces different trends on FGF and SL biases with electron temperature. For increasing values of electron temperature it underestimates the $M_{\text {gas }}$. Considering the FGF approach, this implies an underestimation of the total mass, too. For the SL method this would instead produce an increasing trend in the mass bias with temperature because an $M_{\text {gas }}$ underestimation corresponds to a decreasing gas fraction.

The net result is a mass bias that is always different from zero for the HE approach, as well as for the FGF and SL ones, unless it is for an electron temperature suitable to correctly modeling the CC cluster as an isothermal one but generally different from the true ICM temperature (see the crossovers in mass biases in Fig. 4).

The mass bias reflects a combination of different contributions (Piffaretti \& Valdarnini 2008). In particular for this work, the bias can be reduced to an integration bias and a modeling bias, which are estimated and discussed separately. The integration bias refers to the mass bias due to the different integration ranges along the line of sight. An infinite integration range is considered for the analytic $\Delta T_{\mathrm{SZ}}$ expression, while a finite range is assumed in the numerical projection method, which is limited by the missing knowledge of the electron pressure profile outside the region defined by the data. The integration bias that can be evaluated by still applying the whole procedure to an ISO template (Fig. 4c). The modeling bias reflects the dependence of the bias on the assumed model for the ICM. In order to highlight it and to cancel the integration bias, we apply the procedure to the CC cluster, recovered as an isothermal one. In this case both the cluster signals ( $\mathrm{CC}$ mock data and ISO recovered data) are obtained by projecting the three-dimensional electron pressure profile. For disentangling the integration bias, the ISO profile is also integrated over a limited range along the line of sight. The HE bias ranges between $50 \%$ and $80 \%$, always implying a mass overestimation that is independent of the electron temperature.

\section{Conclusions}

We studied the bias that affects the estimate of cluster total mass by SZ observations when an isothermal beta-model is assumed to describe the ICM physical properties, specifically in the case of $\mathrm{CC}$ clusters when X-ray and/or lensing information is missing.

It is well known that, rather than the central Comptonization parameter $y_{0}$, affected by the choice of cluster profile modeling, an integrated quantity, like the parameter $Y$, appears to be a more robust mass proxy. Nevertheless, we have shown that CC clusters can generate observed $y$ maps in which the ICM morphology could still be substantially hidden, even for the current most sensitive experiments operating from the largest available $\mathrm{mm} /$ submm telescopes.

While a general assumption of cluster morphology is efficient at detecting them in blind SZ maps, the possible mismatch with the actual cluster profile results in a mass bias. In fact simple ICM models, like the isothermal beta-model, applied to SZ observations can wrongly estimate cluster total mass in the presence of peculiar ICM dynamics as in CC clusters, which are studied in the current analysis, and mergers.

We analyzed the mass bias as derived in a limited sample of eight CC clusters observed by Chandra, both nearby 
$(0.1<z<0.5)$ and with high mass $\left(M>10^{14} M_{\odot}\right)$. Under the assumption of an isothermal beta-model, the cluster total mass was derived applying three different approaches: the hydrostatic equilibrium equation, a fixed gas fraction, and a self-similar $M_{\text {tot }}-Y$ relation.

Assuming we had no information from X-ray observations, we reported the bias on the derived total mass as dependent on electron gas temperature. Only in the case of hydrostatic equilibrium does this bias appear almost constant for the considered clusters in the range of 50-80\%. Incidentally, we notice that an electron temperature value exists for which the FGF and SL mass biases vanish. This could be the only case in which a simple isothermal beta-model accurately reproduces the mass of $\mathrm{CC}$ clusters.

The large biases on total cluster mass recovery in CC clusters represent another reason to definitely discard the isothermal beta-model for this purpose and to firmly support more sophisticated models, with universal pressure profiles (e.g. Arnaud et al. 2010). This is already employed for modeling cluster atmospheres in almost all the present blind-survey data reduction (SPT and Planck), and it is planned in the next future for ACT observations.

Acknowledgements. Part of this work has been supported by funding from Ateneo 2009-C26A09FTJ7. We thank S. Borgani and the anonymous referee for their useful comments and suggestions.

\section{Appendix A: Electron temperature profile function for cool core clusters}

Here we describe the simple approach used to obtain the electron temperature profile function applied in this work. The assumptions at the basis of this treatment are

- the knowledge of functional relations of the electron temperature in two different regions of the cluster (see Sect. 2); and

- the approximate location of the maximum of the $T_{\mathrm{e}}$ profile.

Equation (8) can be also written as

$\log \left(\frac{T_{\mathrm{e}}^{\mathrm{CC}}(r)}{T_{\mathrm{x}}}\right)= \begin{cases}m_{1} \log \left(\frac{r}{r_{500}}\right)+q_{1} & \text { for } \frac{r}{r_{500}}<0.1 \\ m_{2} \log \left(\frac{r}{r_{500}}\right)+q_{2} & \text { for } \frac{r}{r_{500}}>0.2\end{cases}$

which is clearly obtained by self-similarity studies of $T_{\mathrm{e}}$ profiles and by assuming a universal $T_{\mathrm{e}}$ profile describing CC clusters. Thus, we need a function that follows those linear trends asymptotically. Following Eq. (A.1) it is simple to associate $A_{1,2}=10^{q_{1,2}}$. For the sake of simplicity, in what follows we call $Y=\log \left(T_{\mathrm{e}}(r) / T_{\mathrm{X}}\right)$ and $X=\log \left(r / r_{500}\right)$.

Though several functions can satisfy these general conditions, we consider the hyperbole as the simplest candidate for yielding a continuum function. It is extremely easy to find the hyperbole equation by knowing its asymptotes, only if they are symmetric with respect to the coordinated axes. In order to be in this simple case, we need to change our reference frame into a more convenient one, which results in translating the old reference frame into the intersections point of the two linear functions and by rotating it by an angle $\alpha_{\mathrm{R}}=$ $0.5\left[\arctan \left(m_{1}\right)+\arctan \left(m_{2}\right)\right]$, which is the angle between the old $X$-axis and the bisectrix of the asymptotes (i.e. the new $X$ axis). The new asymptotes, which are now referred to the new reference frame, are

$Y_{1}^{\mathrm{TR}}=\left[\frac{m_{1} \cos \alpha_{\mathrm{R}}-\sin \alpha_{\mathrm{R}}}{\cos \alpha_{\mathrm{R}}+m_{1} \sin \alpha_{\mathrm{R}}}\right] X=m_{\mathrm{R} 1} X=m_{\mathrm{R}} X$
$Y_{2}^{\mathrm{TR}}=\left[\frac{m_{2} \cos \alpha_{\mathrm{R}}-\sin \alpha_{\mathrm{R}}}{\cos \alpha_{\mathrm{R}}+m_{2} \sin \alpha_{\mathrm{R}}}\right] X=m_{\mathrm{R} 2} X=-m_{\mathrm{R}} X$

where the TR apex indicates that the equations are translated (T) and rotated (R). Now that we have the two symmetric asymptotes with respect to the coordinated axes, we can use the hyperbole equation to build up our function, which reads

$Y^{\mathrm{TR}}= \pm \sqrt{\left(\frac{b}{a}\right)^{2} X^{2}+b^{2}}$

where $a=c / \sqrt{1+m_{\mathrm{R}}^{2}}, b=a m_{\mathrm{R}}$ and $c=\sqrt{a^{2}+b^{2}}$. Since the hyperbole of Eq. (A.4) is now related to the wrong asymptotes, we need to inverse-translate it and inverse-rotate it. The resulting functions are

$$
\begin{aligned}
& Y^{\mathrm{TRR}^{-1}}=\frac{-B B X \pm \sqrt{B B^{2} X^{2}-4 A A\left(C C X^{2}-b^{2}\right)}}{2 A A} \\
& Y^{\mathrm{TRR}^{-1} \mathrm{~T}^{-1}}=\frac{-B B\left(X-X_{P}\right)}{2 A A} \\
& \quad \pm \frac{\sqrt{B B^{2}\left(X-X_{P}\right)^{2}-4 A A\left[C C\left(X-X_{P}\right)^{2}-b^{2}\right]}}{2 A A}+Y_{P}
\end{aligned}
$$

where

$$
\begin{aligned}
& A A=\left[\cos ^{2}\left(\alpha_{\mathrm{R}}\right)-\left(\frac{b}{a}\right)^{2} \sin ^{2}\left(\alpha_{\mathrm{R}}\right)\right] \\
& B B=\left[1+\left(\frac{b}{a}\right)^{2}\right] \sin 2 \alpha_{\mathrm{R}} \\
& C C=\left[\sin ^{2}\left(\alpha_{\mathrm{R}}\right)-\left(\frac{b}{a}\right)^{2} \cos ^{2}\left(\alpha_{\mathrm{R}}\right)\right]
\end{aligned}
$$

and

$$
\begin{aligned}
& X_{P}=\frac{q_{2}-q_{1}}{m_{1}-m_{2}} \\
& Y_{P}=\frac{m_{1} q_{2}-m_{2} q_{1}}{m_{1}-m_{2}} .
\end{aligned}
$$

The parameters $m_{1}, m_{2}, q_{1}, q_{2}$, characterize the asymptotes of the discussed hyperbole. The complete function can be obtained by substituting $(X, Y)$ with $\left(\log \left(r / r_{500}\right), \log \left(T_{\mathrm{e}}(r) / T_{\mathrm{X}}\right)\right)$ and only considering the negative sign. We add a fifth degree of freedom identified with $c$ to the analysis, which gives the focal point position of the hyperbole, and we arbitrarily consider in the analysis $c=0.2$, which is the one that gives a minimum $\chi^{2}$ in the fit with the cluster electron temperature data.

\section{References}

Arnaud, M., Pratt, G. W., Piffaretti, R., et al. 2010, A\&A, 517, A92 Bonamente, M., Joy, M. K., LaRoque, S. J., et al. 2006, ApJ, 647, 25 Bonamente, M., Joy, M. K., LaRoque, S. J., et al. 2008, ApJ, 675, 106 Borgani, S., Murante, G., Springel, V., et al. 2004, MNRAS, 348, 1078 Bryan, G. L., \& Norman, M. L. 1998, ApJ, 495, 80

Cavaliere, A., \& Fusco-Femiano, R. 1978, A\&A, 70, 677

Comis, B., De Petris, M., Conte, A., Lamagna, L., \& De Gregori, S. 2011, MNRAS, submitted 
D’Agostini, G. 2003, Bayesian reasoning in data analysis - A critical introduction (World Scientific Publishing)

De Grandi, S., \& Molendi, S. 2002, ApJ, 567, 163

De Petris, M., Lamagna, L., Luzzi, G., et al. 2007, New Astron. Rev., 51, 368

Eckert, D., Molendi, S., \& Paltani, S. 2011, A\&A, 526, A79

Evrard, A. E., Metzler, C. A., \& Navarro, J. F. 1996, ApJ, 469, 494

Hallman, E. J., Motl, P. M., Burns, J. O., \& Norman, M. L. 2006, ApJ, 648, 852

Hallman, E. J., Burns, J. O., Motl, P. M., \& Norman, M. L. 2007, ApJ, 665, 911

Henning, J. W., Gantner, B., Burns, J. O., \& Hallman, E. J. 2009, ApJ, 697, 1597

Jones, C., \& Forman, W. 1984, ApJ, 276, 38

La Roque, S. 2005, Ph.D. Thesis, the University of Chicago

Lau, E. T., Kravtsov, A. V., \& Nagai, D. 2009, ApJ, 705, 1129

Loken, C., Norman, M. L., Nelson, E., et al. 2002, ApJ, 579, 571

Kaiser, N. 1986, MNRAS, 222, 323

Kay, S. T., da Silva, A. C., Aghanim, N., et al. 2004, Adv. Space Res., 36, 694

Kosowsky, A. 2003, New Astron. Rev., 47, 939

Kravtsov, A. V., Vikhlinin, A., \& Nagai, D. 2006, ApJ, 650, 128

Markevitch, M., Forman, W. R., Sarazin, C. L., \& Vikhlinin, A. 1998, ApJ, 503, 77

Marriage, T. A., Acquaviva, V., Ade, P. A. R., et al. 2010, ApJ, accepted [arXiv: 1010.1065$]$

Masi, S., Battistelli, E., Brienza, D., et al. 2008, Mem. S.A.It., 79, 887

Melin, J. B., Bartlett, J. G., Delabrouille, J., et al. 2011, A\&A, 525, A139

Morandi, A., Ettori, S., \& Moscardini, L. 2007, MNRAS, 379, 518

Mroczkowski, T. 2011, ApJL, 728, 35

Nagai, D., Kravtsov, A. V., \& Vikhlinin, A. 2007, ApJ, 668, 1

Navarro, J. F., Frenk, C. S., \& White, S. D. M. 1995, MNRAS, 275, 720

Nozawa, S., Itoh, N., \& Kohyama, Y. 2005, A\&A, 440, 39

Piffaretti, R., \& Valdarnini, R. 2008, A\&A, 491, 71

Planck Collaboration 2011a, Planck early results 01, The Planck mission, A\&A, accepted, [arXiv:astro-ph/1101.2022]
Planck Collaboration 2011b, Planck early results 11, Calibration of the local galaxy cluster Sunyaev-Zeldovich scaling relations, A\&A, accepted, [arXiv:astro-ph/1101.2026]

Planck Collaboration 2011c, Planck early results 08, The all-sky early Sunyaev-Zeldovich cluster sample, A\&A, accepted,

[arXiv:astro-ph/1101.2024]

Planck HFI Core Team 2011, Planck early results 04, First assessment of the High Frequency Instrument in-flight performance, A\&A, accepted, [arXiv:astro-ph/1101.2039]

Pratt, G. W., Böhringer, H., Croston, J. H., et al. 2007, A\&A, 461, 71

Rasia, E., Ettori, S., Moscardini, L., et al. 2006, MNRAS, 369, 2013

Ruhl, J., Ade, P. A. R., Carlstrom, J. E., et al. 2004, Millimeter and Submillimeter Detectors for Astronomy II, ed. J. Zmuidzinas, W. S. Holland, S. Withington, Proc. SPIE, 5498, 11

Sanderson, A. J. R., Ponman, T. J., \& O’Sullivan, E. 2006, MNRAS, 372, 1496 Sarazin, C. L. 1988, X-ray emission from clusters of galaxies, ed. C. L. Sarazin Sayers, J., Golwala, S. R., Ameglio, S., \& Pierpaoli, E. 2011, ApJ, 728, 39

Sebring, T. A., Giovanelli, R., Radford, S., \& Zmuidzinas, J. 2006, Ground-based and Airborne Telescopes, ed. L. M. Stepp, Proc. SPIE, 6267, 62672C

Sehgal, N., Trac, H., Acquaviva, V., et al. 2011, ApJ, 732, 44

Sunyaev, R. A., \& Zel'dovich, Y. B. 1972, Comments Astrophys. Space Phys., 4, 173

Vanderlinde, K., Crawford, T. M., de Haan, T., et al. 2010, ApJ, 722, 1180

Vikhlinin, A., Markevitch, M., Murray, S. S., et al. 2005, ApJ, 628, 655

Vikhlinin, A., Kravtsov, A., Forman, W., et al. 2006, ApJ, 640, 691

Vikhlinin, A., Burenin, R. A., Ebeling, H., et al. 2009, ApJ, 692, 1060

Voit, G. M. 2005, Rev. Mod. Phys., 77, 207

Zhang, Y. Y., Finoguenov, A., Böhringer, H., et al. 2004, A\&A, 413, 49

Zhang, Y. Y., Finoguenov, A., Böhringer, H., et al. 2007, A\&A, 467, 437

Zhang, Y. Y., Finoguenov, A., Böhringer, H., et al. 2008, A\&A, 482, 451

Zhang, Y. Y., Okabe, N., Finoguenov, A., et al. 2010, ApJ, 711, 1033 